\title{
Flow behaviour of negatively buoyant jets in immiscible ambient fluid
}

\author{
A. Geyer · J. C. Phillips $\cdot$ M. Mier-Torrecilla $\cdot$ \\ S. R. Idelsohn $\cdot$ E. Oñate
}

Received: 5 November 2010/Revised: 29 September 2011/Accepted: 7 October 2011/Published online: 25 October 2011

(C) Springer-Verlag 2011

\begin{abstract}
In this paper we investigate experimentally the injection of a negatively buoyant jet into a homogenous immiscible ambient fluid. Experiments are carried out by injecting a jet of dyed fresh water through a nozzle in the base of a cylindrical tank containing rapeseed oil. The fountain inlet flow rate and nozzle diameter were varied to cover a wide range of Richardson $R i\left(8 \times 10^{-4}<R i<1.98\right)$, Reynolds $\operatorname{Re}(467<R e<5,928)$ and Weber We $(2.40<W e<308.56)$ numbers. Based on the $R e, R i$ and $W e$ values for the experiments, we have determined a regime map to define how these values may control the occurrence of the observed flow types. Whereas Ri plays a stronger role when determining the maximum penetration height, the effect of the Reynolds number is stronger predicting the flow behaviour for a specific nozzle diameter and injection velocity.
\end{abstract}

\section{Introduction}

When a dense fluid is injected vertically upward into a lighter fluid, its momentum is continually being decreased by buoyancy forces until the vertical velocity becomes zero

A. Geyer $(\bowtie) \cdot$ M. Mier-Torrecilla · S. R. Idelsohn · E. Oñate CIMNE International Center for Numerical Models in Engineering, UPC Campus Norte, Edificio C1, C/Gran Capitán s/n, 08034 Barcelona, Spain e-mail: ageyer@ictja.csic.es

\section{A. Geyer}

Institute of Earth Sciences Jaume Almera, CSIC, c/Lluis Solé i Sabaris s/n, 08028 Barcelona, Spain

J. C. Phillips

Department of Earth Sciences, University of Bristol, Wills Memorial Building, Queen's Road, Bristol BS8 1RJ, UK at some finite distance from the source. As the jet reaches its maximum penetration length $h_{\max }$, it reverses its direction and flows back in an annular geometry around the upflow (Fig. 1). Such jets are called negatively buoyant jets or fountains, and the density difference between the ambient and the injected fluids may be due to a variation in either chemical composition or temperature. In this paper, we use the term jet to describe the initial upwards motion and fountain to describe the collapsing dense flow.

Negatively buoyant jets are common both in engineering and in natural science. An everyday example is the ventilation of large open structures such as aircraft hangars, which are heated using ceiling-mounted fans to drive hot air towards the floor. In nature, geophysical buoyant jets resulting from temperature (or salinity) differences can occur in volcanic magma chambers and in the ocean (e.g. Campbell and Turner 1989; Turner and Campbell 1986).

The flow behaviour of negatively buoyant jets may vary depending on the following factors (Cresswell and Szczepura 1993; Papanicolaou and Kokkalis 2008; Turner 1966): (1) jet parameters, (2) environmental parameters, and (3) geometrical factors. The first group of parameters includes the initial jet velocity distribution and turbulence level (whether the jet is laminar or turbulent), as well as the mass, momentum and buoyancy fluxes. The fountain can be described as strong or weak depending on the ratio of buoyancy and momentum flux, or if the fountain is laminar or turbulent. For strong fountains (the discharge momentum is relatively larger than the negative buoyancy of the flow), the fountain top, plunging plume and intrusion flow are distinct features (Fig. 1a). Kinetic energy is converted into potential energy until $h_{\max }$ is reached and then the fluid begins to accumulate at the top of the fountain. As the mass of accumulated fluid increases, eventually the downward buoyancy force exceeds the inertia of the jet and the collapse occurs. 

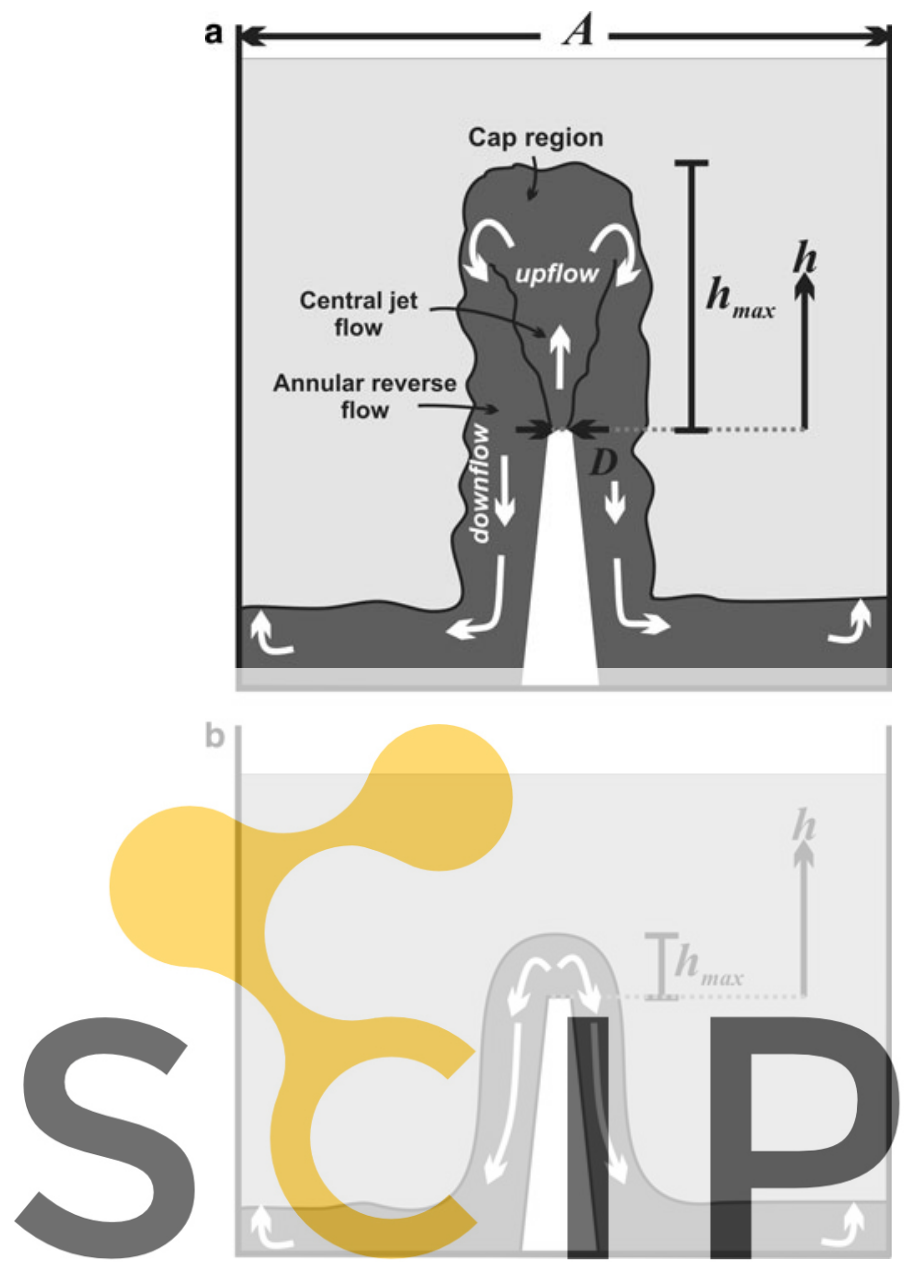

Fig. 1 Schematic diagram of a strong (a) and a weak (b) fountain.

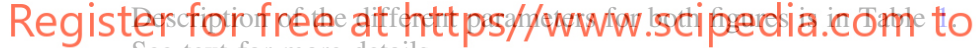
See text for more details

When the falling fluid collapses back to the level of the nozzle, it dislodges from the jet and a new cycle begins. If the source momentum is further increased, this oscillatory behaviour persists at increasing amplitudes until a second threshold limit is reached above which the fountain no longer exhibits high-amplitude pulsations (Clanet 1998). For weak fountains (discharge inertia of the fountains is equal or less than the negative buoyancy force), the fluid exiting the fountain remains attached to the nozzle due to capillary and gravity forces, i.e. the upward and downward flows cannot be visually distinguished. Instead, the streamlines curve and spread from the source and fountain top (Fig. 1b). The second group of variables, environmental parameters, includes parameters describing the ambient fluid (e.g. turbulence level, any net flow, and density stratification) and the geometrical factors include the jet shape, its orientation and proximity to solid boundaries or to the free surface.

Preceding studies suggest that the maximum penetration height $h_{\max }$ of negatively buoyant jets is related to the momentum and buoyancy fluxes at the source and may be expressed in terms of the Richardson $R i$ and Reynolds $R e$ numbers (Armienti et al. 1984; Baines et al. 1990, 1993; Lin and Armfield 2000a, b, 2003; Turner 1966), i.e. $H_{\max } \sim \mathrm{CRi}^{\alpha} \operatorname{Re}^{\beta}$, where $C$ is a constant of proportionality, $\alpha$ and $\beta$ are the scale factors and $H_{\max }$ is the dimensionless maximum penetration height defined as $h_{\max } / D$ (Table 1). Note that the Reynolds number $(R e)$ as defined here characterizes the ratio between inertia and viscous effects in the flow at the nozzle and the Richardson number (Ri) compares gravitational potential energy to kinetic energy (Table 1). After numerous experimental studies, there are significant variations in the reported values of $C$, $\alpha$ and $\beta$ that may be attributed to (List 1982): (1) the methods for defining and measuring the maximum height; (2) the effect of Reynolds number and (3) the effect of relative density difference and of mass flux.

Previous experimental works on negatively buoyant jets considering immiscible ambient-jet fluid pairs have mainly focused on the dynamics of drop formation (Chatterjee and Bradshaw 1972; Meister and Scheele 1696), the estimate of the rise height/jet length (Banks and Chandrasekhara 1963; Friedman 2006; Friedman and Katz 2000; Meister and

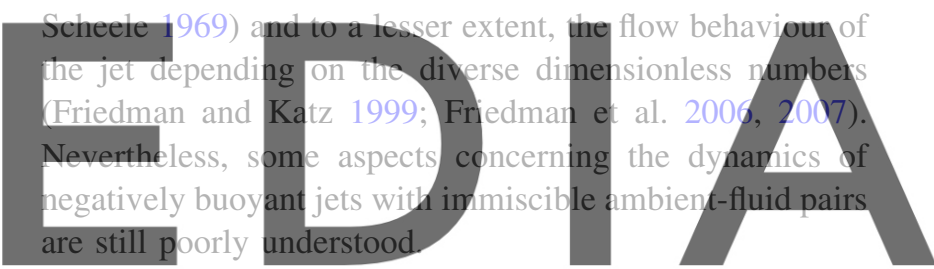

In this paper we investigate experimentally the flow behaviour of a negatively buoyant jet in a homogenous

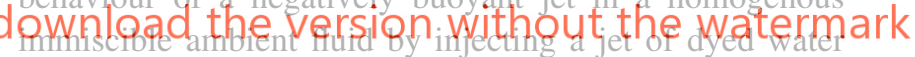

through a nozzle in the base of a cylindrical tank containing

rapeseed oil. One of the main differences between our and previous experiments (Friedman and Katz 1999; Friedman et al. 2006, 2007) (apart from the experimental fluids and their physical properties) is the geometry we are using (Fig. 2): a re-entrant conical nozzle located at the base of the tank whereas in their experiments, they used a bottom issuing fountain (see Fig. 1, Friedman et al. 2006). In the different experiments, we have varied the injection velocity and the nozzle radius to reproduce a wide range of Reynolds, Richardson and Weber numbers. The experiments presented in this paper cover a larger Richardson number interval, $8 \times 10^{-4}<R i<1.98$, than previous studies and are able to reproduce both weak and strong fountains in both turbulent and laminar regimes $(468<\operatorname{Re}<5,928)$. In contrast to previous published results, data obtained allow us to describe three different fountain behaviours (Type I, II and III). Based on the $R e, R i$ and $W e$ values of the numerical and experimental simulations, we present different regime maps to define how $R e, R i$ and $W e$ may control the observed fountain behaviours. 
Table 1 List of the variables and dimensionless numbers referred to in the text

\section{Variables and symbols}

A Container diameter

a Capillary length

$a_{\mathrm{w}} \quad$ Capillary length of water in air (Clanet 1998)

C Constant of proportionality

$D \quad$ Diameter of the nozzle

$D_{\mathrm{p}} \quad$ Width of the lump

$G \quad$ Acceleration due to gravity

$\mathrm{g}^{\prime} \quad$ Reduced gravity

$h_{\max } \quad$ Maximum penetration depth

$Q \quad$ Volumetric flow rate

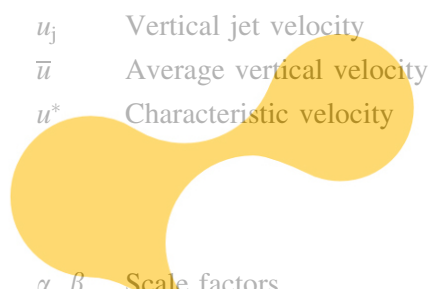

$\alpha, \beta \quad$ Scale factors

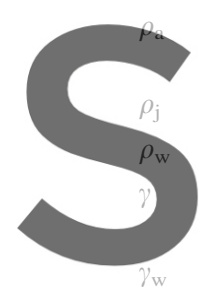

Density of the ambient fluid (rapeseed oil)

Density of the jet fluid (water)

Density of water

Interfacial tension coefficient

(water-rapeseed oil) $^{\mathrm{a}}$

Surface tension of water

Dynamic viscosity of the ambient $200 \times 10^{-3} \mathrm{~Pa} \mathrm{~s}$

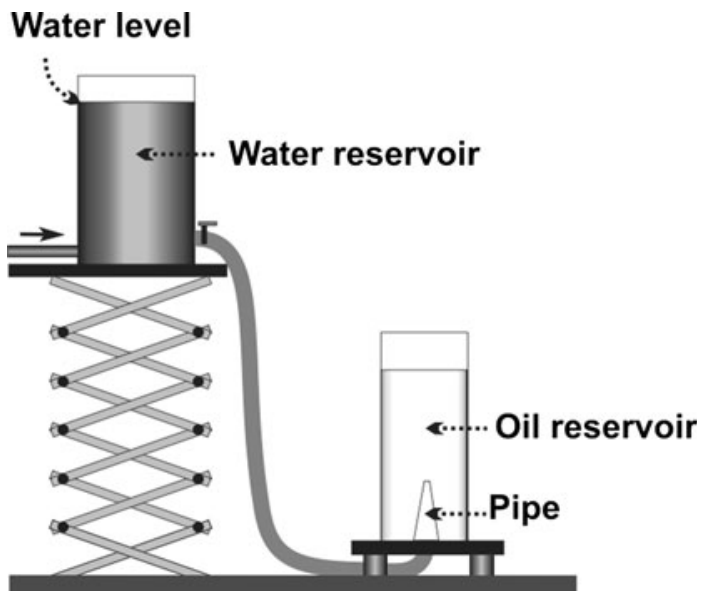

Fig. 2 Schematic diagram of the experimental apparatus

water was injected through a re-entrant conical nozzle with taper angle of $4.5 \pm 0.3^{\circ}$, and the inlet flow rate was kept constant over the duration of the experiment using a constant-head supply tank. The nozzle was situated in the centre of the cylindrical tank, which was $0.1 \mathrm{~m}$ in diameter and $0.3 \mathrm{~m}$ deep, and it was filled to a depth of $0.25 \mathrm{~m}$ with

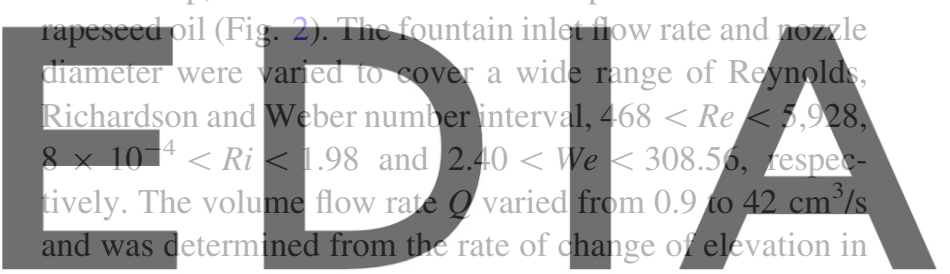

the test chamber. The calculated accuracy of the measurement is $\pm 2,5 \%$. Besides, the nozzle diameter was varied

Register forlfirdenestehtitps//www.scipedia.com to

$\mu_{\mathrm{j}} \quad$ Dynamic viscosity of the injected $10^{-3}, \mathrm{~Pa} \mathrm{~s}$

fluid (water)

Dimensionless numbers

Bo Bond number: buoyancy versus interfacial tension

$\mathrm{Fr} \quad$ Froude number: inertia versus buoyancy

$H_{\max }$ Dimensionless form of $h_{\max }$

Re Reynolds number: inertia versus viscosity

$R i \quad$ Richardson number: buoyancy versus inertia

We Weber number: inertia versus interfacial tension

a Value defined for rapeseed oil and "tap" water at room temperature

\section{Experiments}

Experiments were conducted in which dyed water was injected into the base of a cylindrical tank containing rapeseed oil to form a collapsing fountain (Fig. 2). The
$B o=\frac{\left(\rho_{\mathrm{j}}-\rho_{\mathrm{a}}\right) g D^{2}}{\gamma}=2\left(\frac{D}{a}\right)^{2}$

$F r=\frac{u_{\mathrm{j}}}{\sqrt{D g^{\prime}}}=R i^{-1 / 2}$

$H_{\text {max }}=\frac{h_{\max }}{D}$

$R e=\frac{\rho_{\mathrm{j}} u_{\mathrm{j}} D}{\mu_{\mathrm{j}}}$

$R i=\frac{D g^{\prime}}{u_{\mathrm{j}}^{2}}=F r^{-2}$

$W e=\frac{\rho_{\mathrm{j}} u_{\mathrm{j}}^{2} D}{\gamma}$

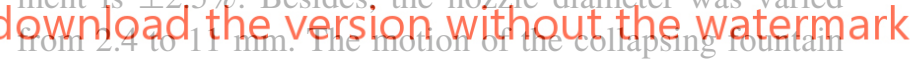

was recorded using a digital camera with resolution in time of less than $0.1 \mathrm{~s}$ and each pixel is $0.001 \times 0.001 \mathrm{~cm}$. The experiments were run for sufficiently short times so that the depth of liquid in the tank, hence the hydrostatic pressure, was not significantly increased.

\section{Results}

3.1 Description of the flow regimes

Our experimental results show that, for a given fountain geometry, the fountain exhibits distinct flow regimes as the inlet volumetric flow rate is increased, as previously observed in other experimental studies (Friedman and Katz 1999; Friedman et al. 2006, 2007). Based on the results obtained, we have been able to categorize three different flow regimes based on the behaviour of the fountain (Fig. 3): Type I, II and III. Flow regime I is characterized by an approximately constant fountain height, within the range of experimental error of the observation (Fig. 3a). In 
Fig. 3 Schematic diagram and photographs of the three different flow types observed in the experiments a

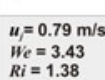
$W_{e}=3.43$ $R i=1.38$
$R e=869$ $R e=869$
Type I

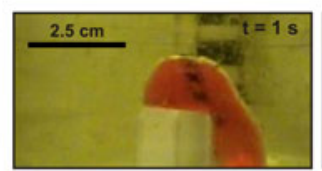

Type II
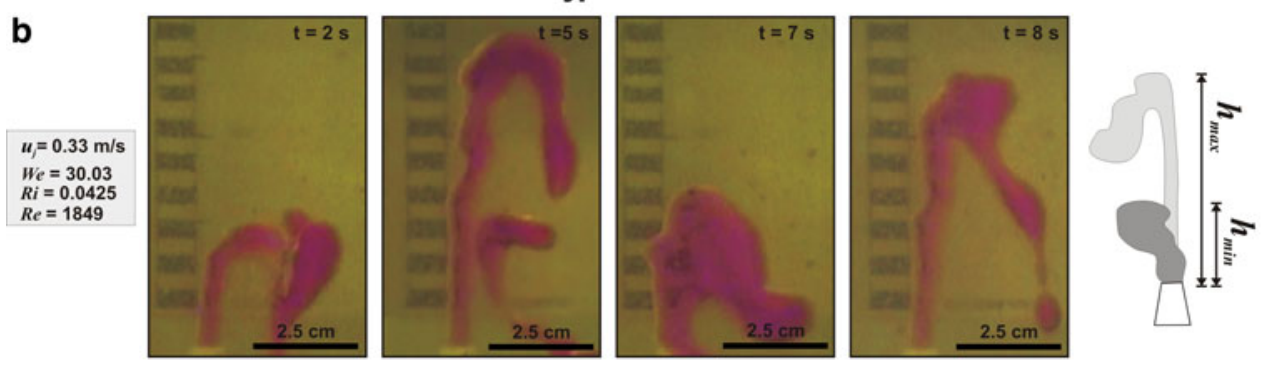

Type III
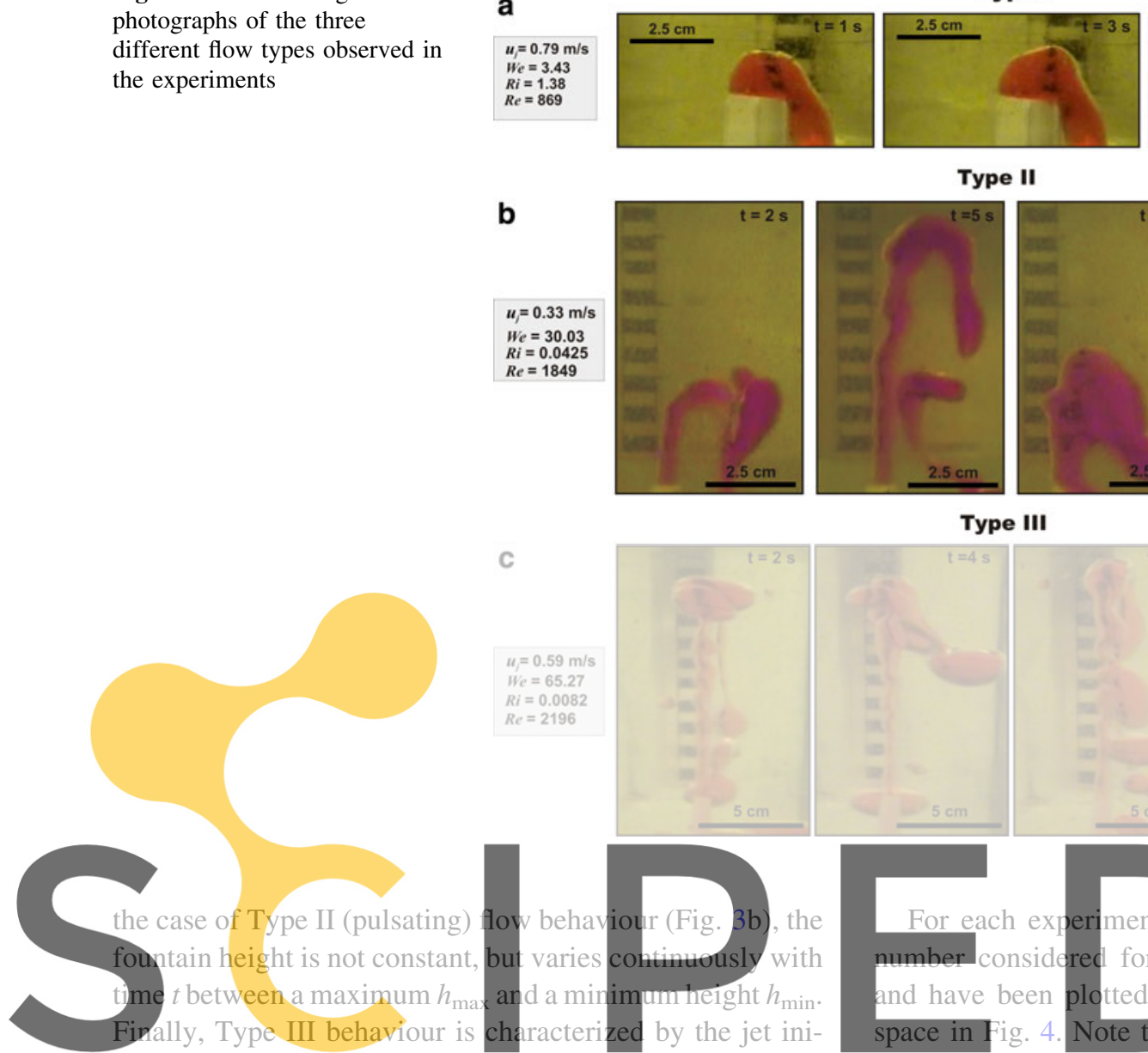

tially penetrating upward into the ambient fluid and when reaching $h_{\max }$, a "cap" of accumulated jet fluid.forms at the

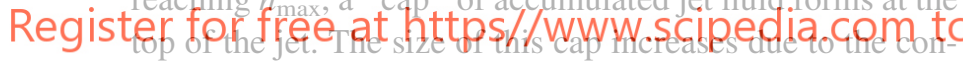
tinuous fluid supply from the fountain, but its vertical position remains constant at $h_{\max }$. Once the cap exceeds a critical size, it breaks up and water droplets fall back to the base of the tank (Fig. 3c). In this regime, the fountain is characterized by a smooth and a wavy part (Fig. 3).

\subsection{Dimensional analysis}

As adopted in various previous publications, dimensional analysis may help to understand and delimit the different flow regimes observed for negatively buoyant jets (e.g. Friedman 2006; Kaye and Hunt 2006). However, an apparently unresolved issue is the choice of length scale to adopt in dimensionless groups (see Table 1). There appears to be general consensus in selecting the width of the nozzle, but some studies use the radius and others the diameter as characteristic lengths (Table 2). This discrepancy is very important when comparing the results obtained from the different studies. Here, we choose the nozzle diameter as the length scale for the flow, on the simple basis that this is the length defined by the solid boundaries of the flow.

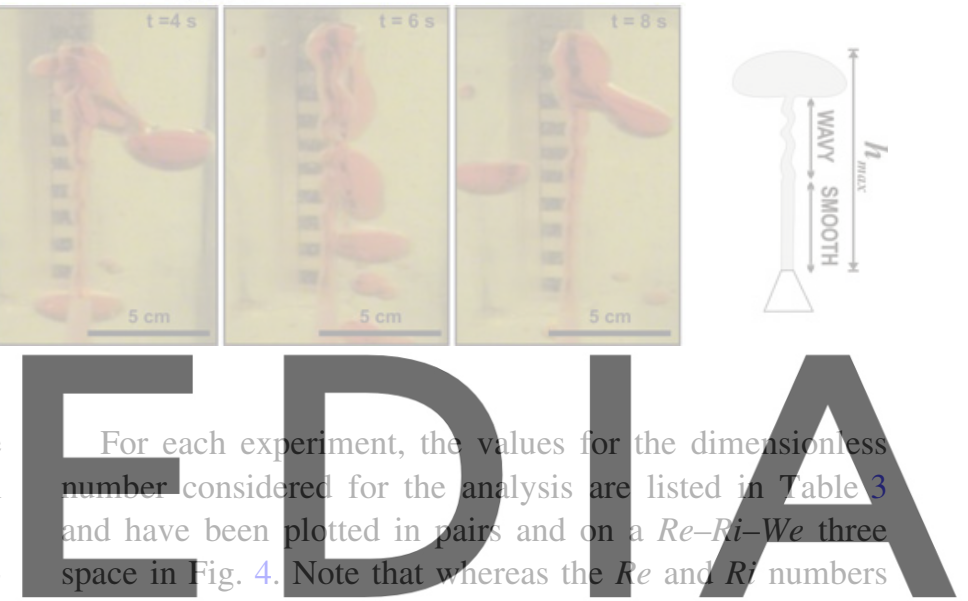

characterize the ratio inertia versus viscous or buoyancy effects, respectively, interfacial tension effects are nondqwelsagizede versved. Witheyt the watermark

For Type I and II behaviour, inertial forces are less important than viscosity or interfacial tension, contrary to the case of Type III experiments for which inertia dominates (Fig. 4). Besides, buoyancy dominates over inertia effects for Type I experiments, but not for Type II and III (Fig. 4b).

From Fig. 4c it is obvious that the transition between Type I and II behaviours to Type III occurs at an approximate constant $W e \approx 35$ (Fig. 4b, c), over $W e>35$ only Type III behaviour is observable. Type I and II are restricted to $W e<35$ and the change from one to the other is mainly controlled by the $R i$ number (Fig. 4c).Type I flow regime is observable for $R i>0.13$ values and below 0.05 only Type II behaviour is observable. In the transition, regime $0.05<R i<0.13$, both Type I or II flow may occur. A more accurate definition of this limit between Type I and II needs further experimental results.

In Fig. 5 we have plotted $H_{\max }$ against $R i$ and $R e$, respectively. There is a clear separation between Type I and III flow regimes at $R i \approx 0.13$, which is not the case 
Table 2 List of most of the experimental works focused on negatively buoyant jets

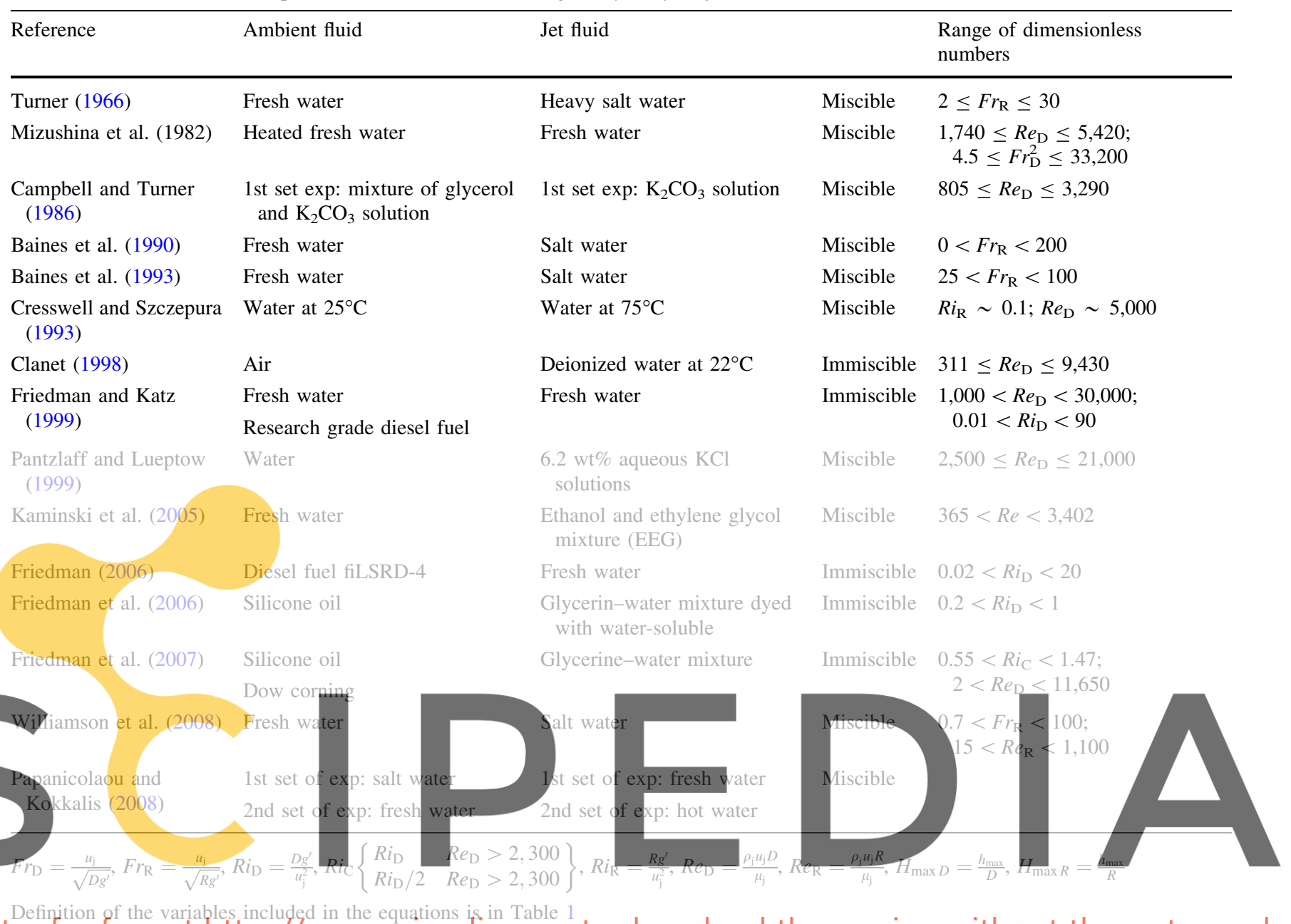

between Type II and III which occur over the same Ri$H_{\text {max }}$ range (Fig. 5a). In their work Friedman and Katz (2000) proposed that three power law relationships are able to explain the penetration depth of a negatively buoyant jet in terms of $R i$ and a jet spreading factor $F$ (see Friedman and Katz 2000 for more details). The paper showed that for $\mathrm{Ri} / \mathrm{F}^{2}<0.2$ the maximum penetration height can be predicted by $H_{\max }=2.2\left(R i / F^{2}\right)^{-0.5}$ and for $R i / F^{2}>0.2$, $H_{\max }=\left(R i / F^{2}\right)^{-1}$. The third power law relationship is applied for $R i / F^{2}>2$ and $1 / D \ll 1$, which is not the case of our experimental results. In their paper Friedman and Katz (2000) stated that these power law correlations fit a wide variety of published data, including miscible and immiscible fluids.

Considering that for the experimental set-up used in this paper $F=1$ (Friedman and Katz 2000), the correlation of our data with $R i<0.2$ is quite consistent with the one proposed by Friedman and Katz (2000) (Fig. 5a), namely: $H_{\text {max }}=2.76(R i)^{-0.45}$. Differences between our correlation and the one proposed by Friedman and Katz (2000) may be related to experimental errors included in both data set. From the results plotted in Fig. 5a is evident that our experiments fit also the power law $H_{\max }=R i^{-1}$ for $R i>0.2$.

Friedman (2006) suggest that, apart from predicting the onset of turbulence, the value of $R e$ has no further effect. However, other authors have seen that the limits of stability are dependent on $R e$ (e.g. Lin and Armfield 2003; Lin and Armfield 2004). This observation is clear in Fig. 5b. All three different flow types can be categorized by values of $H_{\text {max }}$ and $R e$. Thus, it is evident that $R e$ plays a role when describing the different flow behaviours and $R i$ is a key parameter to determine the maximum penetration height.

\section{Discussion}

\subsection{Laminar or turbulence flow}

Whereas Type I and II behaviours occur approximately over the same range of $\operatorname{Re}$ numbers $(467<\operatorname{Re}<2,500)$, 
Table 3 List of the performed experiments with their initial conditions: nozzle diameter $D$, injection velocity $u_{\mathrm{j}}$, and volumetric flow $Q$

\begin{tabular}{|c|c|c|c|c|c|c|c|c|c|c|}
\hline & $h_{\max }(\mathrm{m})$ & $D(\mathrm{~m})$ & $Q\left(\times 10^{-6} \mathrm{~m}^{3} / \mathrm{s}\right)$ & $u_{\mathrm{j}}(\mathrm{m} / \mathrm{s})$ & $H_{\max }$ & $R e$ & $W e$ & $R i$ & Bo & Type \\
\hline EXP-1 & 0.084 & 0.0057 & 8.287 & 0.325 & 14.789 & $1,849.257$ & 30.028 & 0.443 & 1.275 & $\mathrm{I}$ \\
\hline EXP-2 & 0.058 & 0.0090 & 14.661 & 0.230 & 6.466 & $2,072.000$ & 23.875 & 0.549 & 3.178 & I \\
\hline EXP-3 & 0.051 & 0.0090 & 9.477 & 0.149 & 5.684 & $1,339.400$ & 9.977 & 0.161 & 3.178 & $\mathrm{I}$ \\
\hline EXP-4 & 0.014 & 0.0090 & 1.809 & 0.126 & 1.590 & $1,136.462$ & 7.182 & 0.347 & 3.178 & I \\
\hline EXP-5 & 0.011 & 0.0090 & 7.217 & 0.113 & 1.201 & $1,019.950$ & 5.785 & 1.977 & 3.178 & $\mathrm{I}$ \\
\hline EXP-6 & 0.050 & 0.0110 & 22.020 & 0.232 & 4.513 & $2,546.195$ & 29.498 & 1.383 & 4.748 & I \\
\hline EXP-7 & 0.024 & 0.0110 & 14.999 & 0.158 & 2.217 & $1,734.352$ & 13.686 & 0.156 & 4.748 & I \\
\hline EXP-8 & 0.012 & 0.0110 & 6.282 & 0.066 & 1.119 & 726.457 & 2.401 & 0.363 & 4.748 & I \\
\hline EXP-9 & 0.015 & 0.0110 & 7.513 & 0.079 & 1.319 & 868.754 & 3.434 & 0.307 & 4.748 & I \\
\hline EXP-10 & 0.041 & 0.0091 & 13.929 & 0.214 & 4.451 & $1,946.957$ & 20.849 & 0.136 & 3.249 & I \\
\hline EXP-11 & 0.015 & 0.0091 & 9.126 & 0.140 & 1.675 & $1,275.591$ & 8.949 & 0.138 & 3.249 & I \\
\hline EXP-12 & 0.025 & 0.0091 & 9.931 & 0.153 & 2.725 & $1,388.159$ & 10.598 & 0.316 & 3.249 & I \\
\hline EXP-13 & 0.043 & 0.0091 & 14.919 & 0.229 & 4.738 & $2,085.331$ & 23.917 & 0.738 & 3.249 & I \\
\hline EXP-14 & 0.043 & 0.0091 & 14.814 & 0.228 & 4.765 & $2,070.588$ & 23.580 & 0.264 & 3.249 & I \\
\hline EXP-15 & 0.017 & 0.0091 & 9.779 & 0.150 & 1.890 & $1,366.934$ & 10.277 & 0.282 & 3.249 & I \\
\hline EXP-16 & 0.013 & 0.0091 & 6.403 & 0.098 & 1.396 & 895.034 & 4.406 & 0.386 & 3.249 & I \\
\hline EXP-17 & 0.059 & 0.0120 & 21.375 & 0.189 & 4.952 & $2,265.738$ & 21.411 & 0.411 & 5.651 & I \\
\hline EXP-18 & 0.047 & 0.0120 & 20.681 & 0.183 & 3.892 & $2,192.086$ & 20.042 & 0.345 & 5.651 & I \\
\hline EXP-19 & 0.035 & 0.0120 & 17.680 & 0.156 & 2.898 & $1,873.989$ & 14.647 & 0.144 & 5.651 & I \\
\hline EXP-20 & 0.030 & 0.0120 & 17.133 & 0.151 & 2.502 & $1,816.071$ & 13.756 & 0.145 & 5.651 & I \\
\hline 21 & 0.018 & 0.0057 & 2.908 & 0.114 & 3.228 & 648.931 & & 0.002 & 1.275 & I \\
\hline-22 & 0.022 & 0.0057 & 4.495 & 0.176 & 3.945 & $1,002.96$ & & 1.977 & 1.275 & \\
\hline & 0.028 & 0.0057 & 4.488 & 0.176 & & $1,001.50$ & & 0.001 & 1.275 & \\
\hline & 0.062 & 0.0025 & 1.899 & 0.387 & 24.652 & 966.12 & & 0.013 & & \\
\hline-25 & 0.014 & 0.0025 & 0.919 & 0.187 & 5.449 & 467.44 & & & & II \\
\hline-26 & 0.041 & 0.0025 & 0.927 & 0.189 & 16.555 & 471.714 & & 0.010 & & II \\
\hline EXP-27 & 0.069 & 0.0031 & 3.775 & 0.500 & 22.384 & $1,549.018$ & 38.740 & 0.022 & 0.377 & II \\
\hline
\end{tabular}

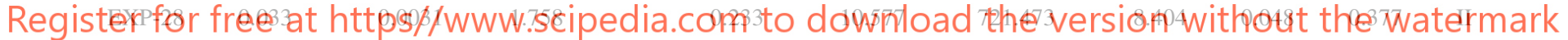

\begin{tabular}{llllrrrrrrrr} 
EXP-29 & 0.071 & 0.0037 & 3.875 & 0.360 & 19.135 & $1,332.295$ & 24.011 & 0.025 & 0.537 & II \\
EXP-30 & 0.056 & 0.0037 & 2.636 & 0.245 & 15.083 & 906.102 & 11.106 & 0.032 & 0.537 & II \\
EXP-31 & 0.072 & 0.0037 & 3.697 & 0.344 & 19.510 & $1,270.862$ & 21.847 & 0.021 & 0.537 & II \\
EXP-32 & 0.055 & 0.0024 & 1.106 & 0.244 & 22.854 & 586.032 & 7.162 & 0.024 & 0.226 & II \\
EXP-33 & 0.049 & 0.0024 & 1.358 & 0.300 & 20.583 & 719.892 & 10.808 & 0.042 & 0.226 & II \\
EXP-34 & 0.053 & 0.0024 & 1.269 & 0.280 & 21.875 & 672.507 & 9.432 & 0.133 & 0.226 & II \\
EXP-35 & 0.025 & 0.0024 & 1.082 & 0.239 & 10.333 & 573.242 & 6.853 & 0.319 & 0.226 & II \\
EXP-36 & 0.077 & 0.0025 & 4.483 & 0.913 & 30.733 & $2,281.128$ & 104.175 & 0.002 & 0.245 & III \\
EXP-37 & 0.073 & 0.0025 & 3.546 & 0.722 & 29.229 & $1,804.193$ & 65.167 & 0.004 & 0.245 & III \\
EXP-38 & 0.097 & 0.0025 & 7.716 & 1.572 & 38.611 & $3,925.895$ & 308.562 & 0.001 & 0.245 & III \\
EXP-39 & 0.078 & 0.0031 & 4.177 & 0.553 & 25.091 & $1,713.840$ & 47.422 & 0.008 & 0.377 & III \\
EXP-40 & 0.078 & 0.0031 & 3.576 & 0.474 & 25.262 & $1,467.122$ & 34.752 & 0.011 & 0.377 & III \\
EXP-41 & 0.081 & 0.0031 & 4.142 & 0.549 & 26.192 & $1,699.651$ & 46.640 & 0.008 & 0.377 & III \\
EXP-42 & 0.093 & 0.0037 & 6.389 & 0.594 & 25.198 & $2,196.486$ & 65.262 & 0.008 & 0.537 & III \\
EXP-43 & 0.103 & 0.0042 & 9.009 & 0.650 & 24.444 & $2,728.361$ & 88.707 & 0.008 & 0.692 & III \\
EXP-44 & 0.068 & 0.0024 & 2.833 & 0.626 & 28.208 & $1,501.503$ & 47.016 & 0.005 & 0.226 & III \\
EXP-45 & 0.079 & 0.0024 & 3.551 & 0.785 & 32.958 & $1,881.802$ & 73.848 & 0.003 & 0.226 & III \\
EXP-46 & 0.076 & 0.0024 & 3.525 & 0.779 & 31.833 & $1,868.361$ & 72.797 & 0.003 & 0.226 & III \\
EXP-47 & 0.079 & 0.0024 & 4.237 & 0.937 & 33.042 & $2,245.363$ & 105.140 & 0.002 & 0.226 & III \\
EXP-48 & 0.082 & 0.0024 & 3.917 & 0.866 & 34.292 & $2,076.027$ & 89.879 & 0.003 & 0.226 & III \\
\hline
\end{tabular}


Table 3 continued

\begin{tabular}{|c|c|c|c|c|}
\hline & $h_{\max }(\mathrm{m})$ & $D(\mathrm{~m})$ & $Q\left(\times 10^{-6} \mathrm{~m}^{3} / \mathrm{s}\right)$ & $u_{\mathrm{j}}(\mathrm{m} / \mathrm{s})$ \\
\hline EXP-49 & 0.078 & 0.0024 & 4.411 & 0.975 \\
\hline EXP-50 & 0.080 & 0.0024 & 3.007 & 0.665 \\
\hline EXP-51 & 0.108 & 0.0091 & 30.639 & 0.471 \\
\hline EXP-52 & 0.077 & 0.0091 & 21.940 & 0.337 \\
\hline EXP-53 & 0.066 & 0.0091 & 19.770 & 0.304 \\
\hline EXP-54 & 0.076 & 0.0091 & 24.659 & 0.379 \\
\hline EXP-55 & 0.078 & 0.0091 & 21.136 & 0.325 \\
\hline EXP-56 & 0.096 & 0.0091 & 26.711 & 0.411 \\
\hline EXP-57 & 0.125 & 0.0091 & 42.412 & 0.652 \\
\hline EXP-58 & 0.103 & 0.0091 & 26.106 & 0.401 \\
\hline EXP-59 & 0.086 & 0.0120 & 33.322 & 0.295 \\
\hline EXP-60 & 0.099 & 0.0120 & 33.009 & 0.292 \\
\hline EXP-61 & 0.074 & 0.0120 & 35.081 & 0.310 \\
\hline EXP-62 & 0.104 & 0.0120 & 36.320 & 0.321 \\
\hline EXP-63 & 0.058 & 0.0120 & 27.590 & 0.244 \\
\hline EXP-64 & 0.096 & 0.0057 & 9.087 & 0.356 \\
\hline EXP-65 & 0.067 & 0.0057 & 8.196 & 0.321 \\
\hline EXP-66 & 0.112 & 0.0057 & 11.654 & 0.457 \\
\hline EXP-67 & 0.102 & 0.0057 & 9.230 & 0.362 \\
\hline EXP-68 & 0.154 & 0.0057 & 19.751 & 0.774 \\
\hline & 0.139 & 0.0057 & 14.982 & \\
\hline EXP-70 & 0.123 & 0.0057 & 13.580 & 532 \\
\hline
\end{tabular}

$(1,500<\operatorname{Re}<5,928)$ (Table 3). An important issue is

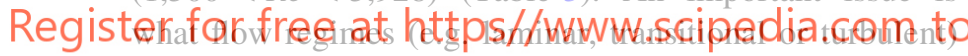

these ranges of $R e$ pertain to for a negatively buoyant jet. For example, in the case of pipe flow, the transition region is approximately in the interval $2,000<R e<4,000$, so if these limits were appropriate for a negatively buoyant jet, our experimental $R e$ values would correspond principally to a laminar but also partially a transitional regime (for Type I and II) and for Type I mainly to the transitional but also the turbulent regime (Table 3).

However, previous studies of negatively buoyant jets have established no consensus as to the regime delimiting values of $R e$. Pearce (1966) on the basis of visual observations using dye of the structure of nearly non-buoyant jets over a Reynolds number range of 68-13,100, establishing in general terms that the jet was essentially laminar for $R e<500$ and fully turbulent for $R e>3,000$. Values of $R e$ in between lead to a transitional regime where a part of the jet behaved as laminar and the other as turbulent. Another interesting classification is that proposed by Williamson et al. (2008), who established the laminar-transitional threshold at $R e=240$ and the transitional-turbulent at $R e=4,000$. Considering both Pearce (1966) and

\begin{tabular}{|c|c|c|c|c|c|}
\hline$H_{\max }$ & $R e$ & $W e$ & $R i$ & Bo & Type \\
\hline 32.417 & $2,337.664$ & 113.961 & 0.002 & 0.226 & III \\
\hline 33.208 & $1,593.532$ & 52.956 & 0.004 & 0.226 & III \\
\hline 11.905 & $4,282.635$ & 100.875 & 0.032 & 3.249 & III \\
\hline 8.449 & $3,066.735$ & 51.727 & 0.063 & 3.249 & III \\
\hline 7.237 & $2,763.381$ & 42.000 & 0.077 & 3.249 & III \\
\hline 8.297 & $3,446.699$ & 65.339 & 0.050 & 3.249 & III \\
\hline 8.620 & $2,954.277$ & 48.003 & 0.068 & 3.249 & III \\
\hline 10.584 & $3,733.516$ & 76.665 & 0.042 & 3.249 & III \\
\hline 13.736 & $5,928.132$ & 193.285 & 0.017 & 3.249 & III \\
\hline 11.334 & $3,648.945$ & 73.231 & 0.044 & 3.249 & III \\
\hline 7.164 & $3,532.080$ & 52.034 & 0.109 & 5.651 & III \\
\hline 8.241 & $3,498.820$ & 51.058 & 0.111 & 5.651 & III \\
\hline 6.140 & $3,718.500$ & 57.671 & 0.098 & 5.651 & III \\
\hline 8.641 & $3,849.866$ & 61.818 & 0.091 & 5.651 & III \\
\hline 4.855 & $2,924.436$ & 35.670 & 0.158 & 5.651 & III \\
\hline 16.875 & $2,027.870$ & 36.109 & 0.035 & 1.275 & III \\
\hline 11.671 & $1,828.864$ & 29.369 & 0.043 & 1.275 & III \\
\hline 19.663 & $2,600.517$ & 59.381 & 0.021 & 1.275 & III \\
\hline 17.829 & $2,059.717$ & 37.252 & 0.034 & 1.275 & III \\
\hline 27.018 & $4,407.505$ & 170.575 & 0.007 & 1.275 & III \\
\hline 24.342 & $3,343.373$ & & 0.013 & 1.275 & \\
\hline 21.518 & $3,030.47$ & & 0.016 & 1.275 & \\
\hline
\end{tabular}

Williamson et al. (2008) definitions, all Type I and I

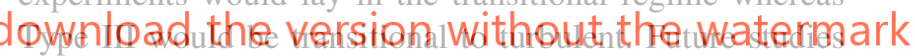
need to investigate this aspect in detail and to try to define the limits for the laminar and turbulent regime for negatively buoyant jets in immiscible fluids since it can provide an important control on the mixing process of both fluids as suggested by Friedman et al. (2006).

\section{2 "Stable" versus "unstable" regime}

Our experimental results, in agreement with previous published studies (Friedman and Katz 1999; Friedman et al. 2006, 2007), show that for a given fountain geometry and ambient-jet fluid pair, the fountain behaviour transitions through distinct flow regimes as the volumetric flow rate (i.e. the vertical jet velocity) increases. Friedman et al. (2006, 2007) suggest that the most significant transition (referred as the "instability threshold", IT) occurs when the flow pattern passes from a "stable" regime where the buoyancy, interfacial tension and viscosity dominate, to an "unstable" regime where momentum dominates. The stable flow regime is characterized by a low, wide and rapidly collapsing fountain, while the unstable flow regime 

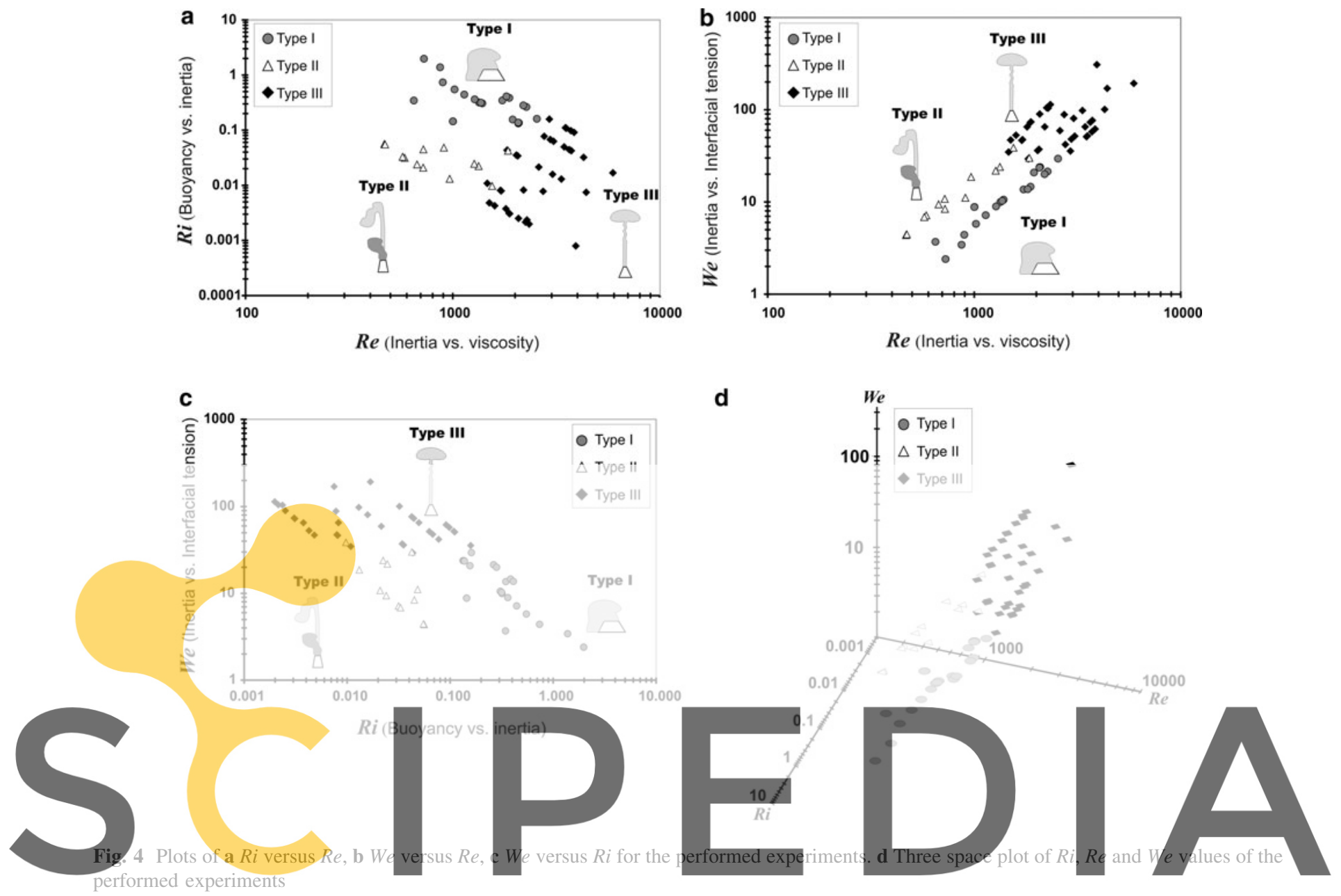

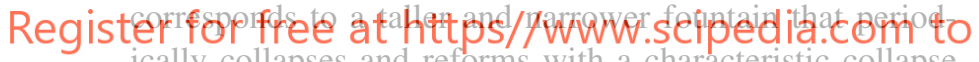 ically collapses and reforms with a characteristic collapse} frequency. Friedman and co-workers (Friedman 2006;

Friedman and Katz 1999; Friedman et al. 2006, 2007) define the IT using only the Richardson number and suggest that the transition between regimes occurs at approximately $R i_{\mathrm{IT}}=1$ for turbulent flow and $R i_{\mathrm{IT}}=2$ for laminar flow.

It is important to note that Friedman et al. (2006) suggested that the $R e$ dependency can be eliminated by defining $R i$ in terms of the characteristic velocity $\left(u^{*}\right)$, which is representative of the momentum of the flow. For turbulent flows, with a nearly uniform velocity profile, the characteristic jet velocity is approximately equal to the volumetric flow rate $Q$ divided by the cross-sectional area of the source $\left(u^{*} \cong \bar{u}=\frac{4 Q}{\pi D^{2}}\right)$, while for laminar flows, the characteristic velocity is defined as the root mean square velocity $\left(u^{*}=\bar{u} \sqrt{2}\right)$ to account for additional momentum (Friedman et al. 2006) The effect of $R e$ may be incorporated by defining a corrected Richardson number $\left(R i_{\mathrm{C}}\right)$. For the turbulent regime (nominally $R e>2,300$ ), $R i_{\mathrm{C}}=R i$ and $R i_{\mathrm{C}}=R i / 2$ for the laminar regime $(R e<2,300)$. In this

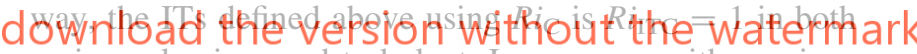 regimes, laminar and turbulent. In common with previous studies (Lin and Armfield 2000a, b; Lin and Armfield} 2003, 2004), we consider necessary to independently analyse the dependence of the flow on $R e$ and $R i$, and therefore, we have not corrected $R i$.

Whereas Friedman et al. (2006, 2007) propose only a two end-member classification as "stable" and "unstable" jets, our experiments identify three distinct flow regimes: Type I, II and III. In the broadest sense, our Type I experiments correspond to the "stable" regime, and our Type II and III experiments exhibit periodic collapsing, which would correspond both to "unstable" flows. However, there is a clear distinction between the collapse mechanism for Type II and III experiments that is not captured in the simple classification proposed by Friedman and co-workers (Friedman 2006; Friedman and Katz 1999; Friedman et al. 2006, 2007). Although some fluctuations of the column are observed in Type III flows, they are not related to a collapse of the fountain as is the case of the jets in Type II regime but to the growth and breakup of the cap region. 

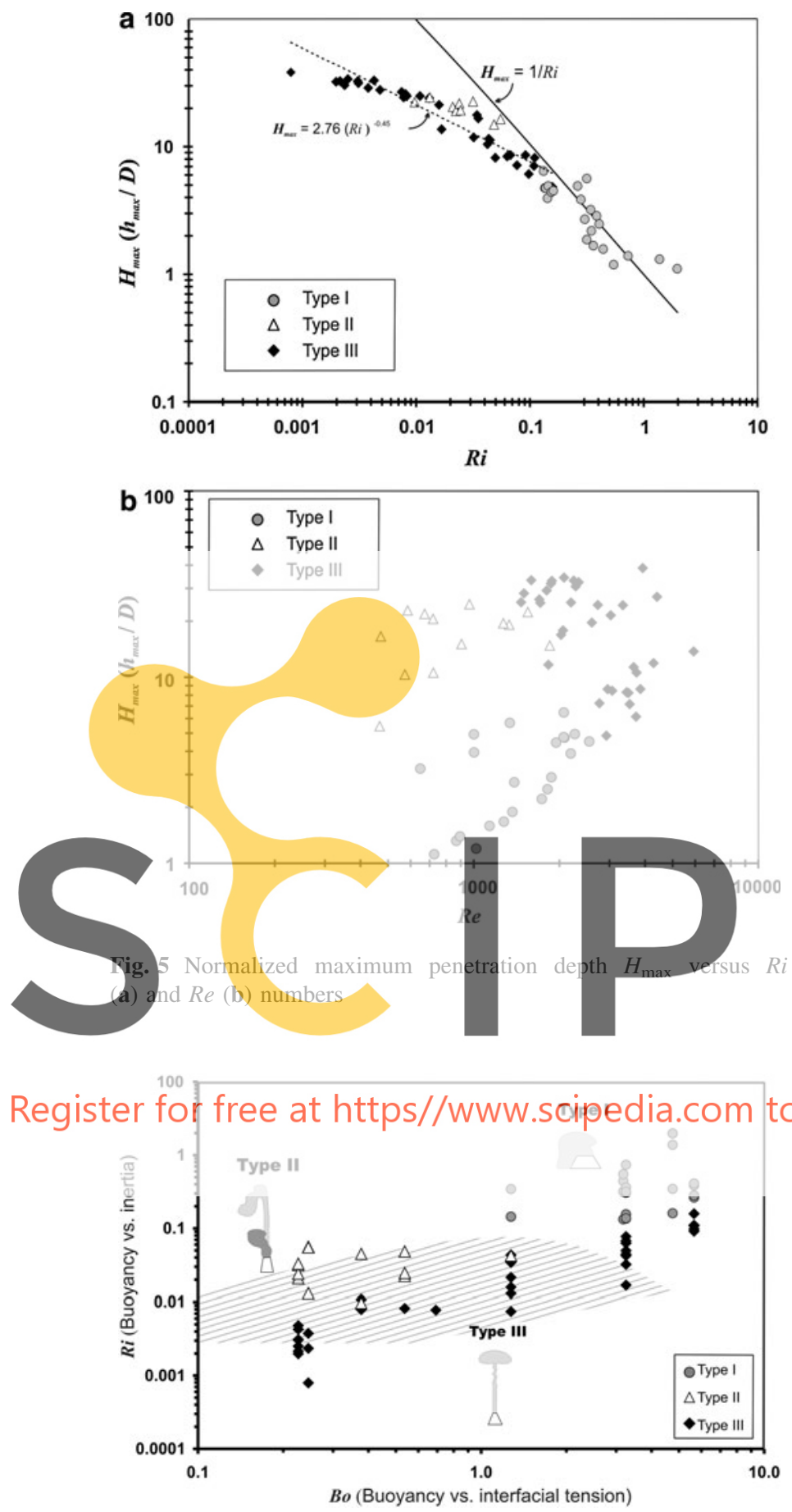

Fig. 6 Plot of $R i$ versus $B o$ for the performed experiments. The dashed area corresponds to the existence limit of the pulsating regime defined by Clanet (1998)

Additionally, the IT defined at $R i_{\mathrm{IT}}=1$ for turbulent flow and $R i_{\mathrm{IT}}=2$ for laminar flow is not directly applicable to our results. According to Fig. 4a, our IT (transition between Type I and II) occurs at $R i \approx 0.13$. However, the discrepancy between the $R i$ values for IT may be due to the high interfacial tension between oil and water. The critical
Richardson number $R i_{\mathrm{IT}}$ has been shown to depend on the interfacial tension $\gamma$ between both fluids and the viscosity ratio $\mu_{\mathrm{j}} / \mu_{\mathrm{a}}$ (Friedman et al. 2007). Interfacial tension contributes to the stability of the fountain and thus decreases $R i_{\mathrm{IT}}$. Viscosity ratios deviating from unity also stabilize the fountain, inhibit the formation of waves on the interface, and delay or even suppress the jet breakup into droplets (Campbell and Turner 1989). In our experiments, $\mu_{\mathrm{j}} / \mu_{\mathrm{a}}=$ 0.005 , outside the range of values considered by Friedman et al. (2007), but their results indicate that $R i_{\text {ITC }}$ may decrease from 1 to 0.7 when reducing viscosity ratio from 1 to $\mu_{\mathrm{j}} / \mu_{\mathrm{a}}=0.2$. Considering the differences in viscosity ratios between our study and that of Friedman et al. (2007), we do not expect agreement on the values of $R i_{\mathrm{IT}}$.

Clanet (1998) presented results from a study of a water jet injected vertically upwards into air which showed that depending on the initial momentum flux $\left(\sim \rho u_{j}^{2}\right)$, water fountains exhibit distinct modes of behaviour. For very low-momentum fluxes, the water exiting the fountain remains attached to the nozzle due to capillary and gravity forces (Dias and Vanden-Broeck 1990). For values of the momentum flux above a certain threshold, a second regime is achieved where the fluid detaches from the nozzle,

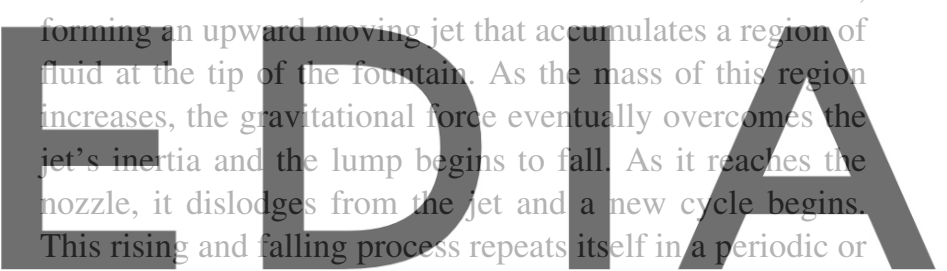

quasi-periodic fashion resulting in large-amplitude oscillations in the fountain height. As the water momentum flux

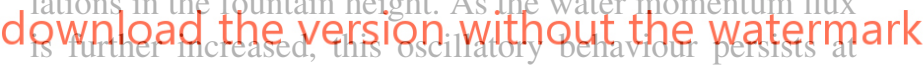

increasing amplitudes until a second threshold limit is reached above which the fountain no longer exhibits highamplitude pulsations. According to his description, Clanet (1998) also observes three flow regimes controlled primarily by the momentum flux (expressed in dimensionless form as the $R i$ number).

In two of the three experimental studies available for the injection of a negatively buoyant jet in an immiscible ambient fluid (Clanet (1998) and our experiments) three flow regimes are observable. By contrast, in their experiments, Friedman et al. $(2006,2007)$ are able to observed only two flow regimes. These differences can be explained due to differences in the experimental geometry and the physical properties of the fluids used. Friedman et al. (2006, 2007) used a nozzle whose exist was located at the same level as the base of the tank. By contrast, our study and that of Clanet (1998) use a re-entrant nozzle whose exit is located away from the solid boundary of the tank. In addition, the diameters of the nozzles used varied from less than one millimetre (Clanet 1998) to several centimetres (Friedman et al. 2006, 2007). Whereas our range of 
$D$ values $(2.4-11 \mathrm{~mm})$ allows us to observe flow behaviours characteristic for narrow to intermediate size nozzles, Friedman et al. $(2006,2007)$ and Clanet (1998) observations are restricted to large and narrow nozzles, respectively. Comparing the $D$ values used by Clanet (1998) $(0.318-4.1 \mathrm{~mm})$ with our own and noting that both experiments use water as the injected fluid, we would expect some similarity of qualitative observations. However, since the ambient fluid is not the same in both cases (Clanet (1998) injects water into air), results obtained are slightly different as explained in the next section.

\subsection{Type II behaviour: the "pulsating" regime}

Following the work of Clanet (1998) we now consider the region of existence of the Type II “pulsating” regime According to Clanet (1998), the pulsating mode starts once the jet momentum flux is high enough to overcome capillary and gravity forces. There are two different mechanisms leading to the threshold between Type II and III (end of the pulsating regime). The first originates in the capillary instability of the Rayleigh-type undergone by the cylindrical jet, which is unstable with respect to disturbances of
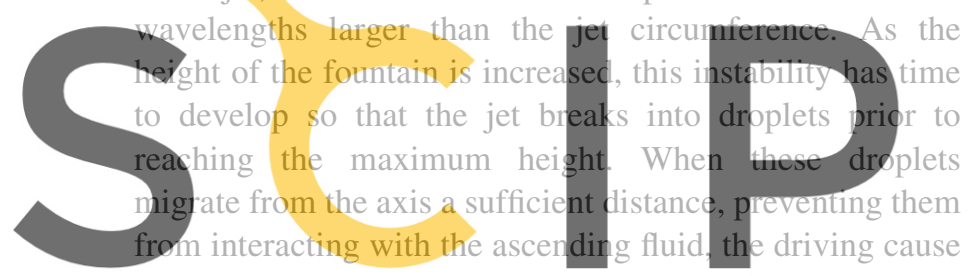

of the oscillation is lost and the fountain exhibits a quasiconstant height, close to its maximum height. $h_{\max }$. If the

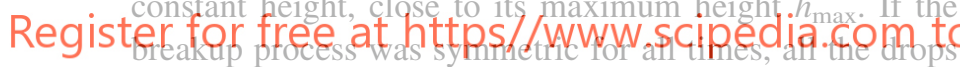
would stay on the axis of symmetry and the oscillations would persist independently of the Rayleigh instability. However, as the jet breaks up, the drops acquire a small radial velocity. When the drops have time to migrate a distance of the order of the jet diameter $D$ before they reach $h_{\max }$, the oscillations stop. An additional physical phenomenon affecting the stability of large-diameter fountains occurs when the dynamic pressure of the jet $\sim \rho u_{\mathrm{j}}^{2}$, becomes of the same order of magnitude as the surface tension restoring action, $\sim 4 \gamma / D_{\mathrm{p}}$ being $D_{\mathrm{p}}$ the width of the lump (Clanet 1998; Taylor 1963). In this limit, the region of accumulated liquid at the fountain topbursts close to its maximum height and no large-amplitude oscillations are observed.

In the case of water fountains, the pulsating regime exists within the limits $0.63 \leq a_{\mathrm{w}} / D \leq 10$ and $20 \leq u_{\mathrm{j}}^{2} /$ $g D \leq 400$, where $a_{\mathrm{w}}$ is the capillary length of water in air defined as (Clanet 1998): $a_{\mathrm{w}}=\left(2 \gamma_{\mathrm{w}} /\left(\rho_{\mathrm{w}} g\right)\right)^{1 / 2}, \rho_{\mathrm{w}}$ and $\gamma_{\mathrm{w}}$ being density and surface tension of water, respectively (Table 1). Notice that $u_{\mathrm{j}}{ }^{2} / g D$ is a reciprocal of the Richardson number with the reduced gravity removed and $a_{\mathrm{w}} / D$ is directly related to a Bond number for the experiment considering that the characteristic length is the nozzle diameter (Table 1). Thus, we have analysed the range of our data in terms of the latter dimensionless numbers $R i$ and $B o$ and compared them with the existence domain for the large-amplitude oscillating fountains defined by Clanet (1998) (Fig. 6).

From Fig. 6 we observe that several of the experiments where Type II (pulsating) behaviour has been observed fall into the region of existence of the pulsating regime observed for water fountains in air (Clanet 1998). However, also some of our Type I experiments fall into the pulsating regime defined by Clanet (1998). A simple explanation for this observation is the fact that we are using a different ambient fluid, i.e. rapeseed oil instead of air. For our experimental configuration we estimate the capillary length of water into rapeseed oil correcting the definition of Clanet (1998) with the density difference between both fluids $a=\left(2 \gamma /\left(\left(\rho_{\mathrm{j}}\right.\right.\right.$ $\left.\left.\left.\rho_{\mathrm{a}}\right) g\right)\right)^{1 / 2}$ (e.g. Aarts 2005) (Table 1). A simple calculus using the values for $\gamma_{\mathrm{w}}, \gamma, \rho_{\mathrm{w}}, \rho$ and $g$ listed in Table 1 allows us to identify that $a_{\mathrm{w}} \approx 0.54 a$, i.e. according to the definition of the Bond number used in this work (Table 1) the Bo values for the immiscible ambient-jet fluid pair are around three

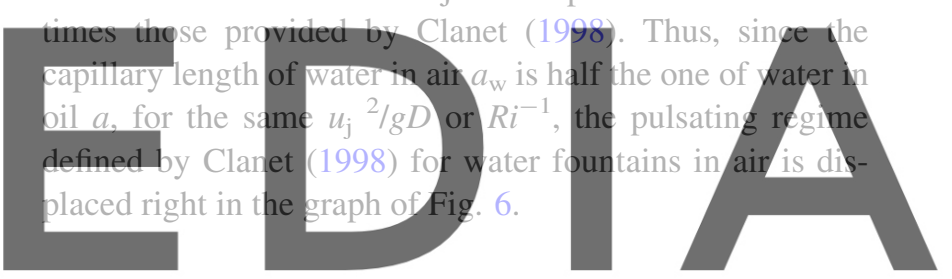

\section{dôwinhoad the quepsifons without the watermark}

In this study we have investigated experimentally the dynamics of negatively buoyant jets in a homogenous immiscible ambient fluid. Experiments are carried out by injecting coloured water into a cylindrical tank containing rapeseed oil. The water is injected using a re-entrant trimmed conical nozzle and maintained at a constant flow rate throughout the experiment. The fountain inlet flow rate and nozzle diameter were varied to cover a wide range of Reynolds, Richardson and Weber number interval, $468<\operatorname{Re}<5,928,8 \times 10^{-4}<R i<1.98$ and $2.40<$ $W e<308.56$, respectively.

In contrast to many previous published studies that propose two end-member classifications of fountain behaviour: "stable" and "unstable", our experimental results show three distinct flow regimes:

- Type I behaviour is characterized as very stable. The height of the fountain is approximately constant although we cannot discount very small fluctuations of the column height within the systematic measurement error. 
- Type II behaviour is described as a pulsating fountain for which height oscillates continuously with time from a maximum $h_{\max }$ to a minimum height $h_{\min }$.

- Type III behaviour is observable for higher injection velocities. The jet initially penetrates upward into the ambient fluid and when it reaches $h_{\max }$, a "cap" forms at the top of the jet. The fountain is characterized by a smooth and a wavy part.

Based on the $R e, R i$ and $B o$ values for the experimental simulations, we have determined a regime map to define how these values may control the occurrence of each of the observed flow types. We find that $R i$ may play a stronger role compared to $R e$ to determine the penetration of the maximum penetration height. By contrast, the effect of the Reynolds and We numbers may be stronger than Ri's to provide a prediction of the flow behaviour for a specific nozzle diameter and injection velocity. The transition between Type I and II is uniquely controlled by the $R i$ number and there is a clear control of the Weber number when passing from Type I or II to Type III.

The region of existence of the Type II (pulsating) regime coincides with the one observed for water fountains in air (Clanet 1998). The main difference is due to the fact that the capillary length of water in air is half the one of water in rapeseed oil.

Acknowledgments M. Mier-Torrecilla thanks the Catalan Agency for Administration of University and Research Grants (AGAUR), the European Social Fund and CIMNE for their support. AG is grateful for her post-doctoral Beatriu de Pinós Grant (2008 BP B 00318) and her Juan de la Cierva Grant (JCI-2010-06092). We thank three anonymous reviewers for their interesting comments that have helped us to improve the previous version of this manuscript. This work was partially supported by the European Research Council under the Advanced Grant: ERC-2009-AdG "Real Time Computational Mechanics Techniques for Multi-Fluid Problems".

\section{References}

Aarts DGA (2005) Capillary length in a fluid-fluid demixed colloidpolymer mixture. J Phys Chem 109:7407-7411

Armienti P, Barberi F, Innocenti F (1984) A model of the Phlegraean Fields magma chamber in the last 10,500 years. Bull Volcanol 47:349-358

Baines WD, Corriveau AF, Reedman TJ (1990) Turbulent fountains in a open chamber. J Fluid Mech 212:557-592

Baines WD, Corriveau AF, Reedman TJ (1993) Turbulent fountains in a closed chamber. J Fluid Mech 255:621-646

Banks RB, Chandrasekhara S (1963) Experimental investigation of the penetration of a high-velocity gas jet through a liquid surface. J Fluid Mech 15:13-34

Campbell IH, Turner JS (1986) The influence of viscosity on fountains in magma chambers. J Petrol 27:1-30

Campbell IH, Turner JS (1989) Fountains in magma chambers. J Petrol 30:885-923
Chatterjee A, Bradshaw AV (1972) Breakup of a liquid surface by an impinging gas jet. J Iron Steel Inst 210:179-187

Clanet C (1998) On large-amplitude pulsating fountains. J Fluid Mech 366:333-350

Cresswell RW, Szczepura RT (1993) Experimental investigation into a turbulent jet with negative buoyancy. Phys Fluids 5:2865-2878

Dias F, Vanden-Broeck J-M (1990) Flows emerging from a nozzle and falling under gravity. J Fluid Mech 213:465-477. doi: $10.1017 /$ S0022112090002403

Friedman PD (2006) Oscillation in height of a negatively buoyant jet. Trans ASME 128:880-882

Friedman PD, Katz J (1999) The flow and mixing mechanisms caused by the impingement of an immiscible interface with a vertical jet. Phys Fluids 11:2598-2606

Friedman PD, Katz J (2000) Rise height for negatively buoyant fountains and depth of penetration for negatively buoyant jets impinging an interface. ASME J Fluids Eng 122:779-782

Friedman PD, Meyer WJ Jr, Carey S (2006) Experimental simulation of phase mingling in a subaqueous lava fountain. J Geophys Res 111:B07201

Friedman PD, Vadakoot VD, Meyer WJ Jr, Carey S (2007) Instability threshold of a negatively buoyant fountain. Exp Fluids 42:751-759

Kaminski E, Tait S, Carazzo G (2005) Turbulent entrainment in jets with arbitrary buoyancy. J Fluid Mech 526:361-376

Kaye NB, Hunt GR (2006) Weak fountains. J Fluid Mech 558:319-328

Lin W, Armfield SW (2000a) Very weak fountains in a homogeneous fluid. Numer Heat Transf Part A 38:377-396

Lin W, Armfield SW (2000b) Direct simulation of weak axisymmetric fountains in a homogeneous fluid. J Fluid Mech 403:67-88

Lin W, Armfield SW (2003) The Reynolds and Prandtl number dependence of weak fountains. Comput Mech 31:379-389

Lin W, Armfield SW (2004) Direct simulation of fountains with intermediate Froude and Reynolds number. ANZIAM J 45:C66C77

List EJ (1982) Turbulent jets and plumes. Annu Rev Fluid Mech 14:189

Meister BJ, Scheele GF (1696) Drop formation from cylindrical jets in immiscible liquid systems. AIChE J 15:700-706

Meister BJ, Scheele GF (1969) Prediction of jet length in immiscible liquid systems. AIChE J 15:689-699

Pantzlaff L, Lueptow RM (1999) Transient positively and negatively buoyant turbulent round jets. Exp Fluids 27:117-125

Papanicolaou PN, Kokkalis TJ (2008) Vertical buoyancy preserving and non-preserving fountains in a homogeneous calm ambient. Int J Heat Mass Transf 51:4109-4120

Pearce AF (1966) Critical Reynolds number for fully-developed turbulence in circular submerged water jets, vol MEG 475, Pretoria, South Africa

Taylor GI (1963) The shape and acceleration of a drop in a high-speed air stream. Scientific papers, vol III. Cambridge University Press, Cambridge, p 457

Turner JS (1966) Jets and plumes with negative or reversing buoyancy. J Fluid Mech 26:779-792

Turner JS, Campbell IH (1986) Convection and mixing in magma chambers. Earth Sci Rev 23:255-352

Williamson N, Srinarayana N, Armfield SW, McBain G, Lin W (2008) Low-Reynolds-number fountain behaviour. J Fluid Mech 608:297-317 